\title{
Red Giant Stars in Magellanic Cloud Clusters: Constraining Population Synthesis Models
}

\author{
Léo Girardi \\ Max-Planck-Institut für Astrophysik, Garching bei München, Germany
}

\begin{abstract}
We have developed a population synthesis code, aimed mainly at the interpretation of CMDs. Magellanic Cloud clusters have been largely used for the test/calibration of the underlying stellar tracks.
\end{abstract}

We computed a large set of stellar evolutionary tracks (Girardi et al. 1999), replacing the Bertelli et al.'s (1994) database for $M \leq 7 M_{\odot}$. The mass resolution has been improved, and the input physics updated. The complete mass range goes from 0.15 to $120 M_{\odot}$. The new sets are available for $Z=0.001,0.004,0.008$, $0.019,0.03$, and more are in progress. From these tracks, theoretical isochrones are constructed for any intermediate value of age and $Z$.

Some critical checks have been performed using Magellanic Cloud clusters:

The luminosity of clump stars presents a minimum at ages of $\sim 1 \mathrm{Gyr}$, just prior to the onset of degenerate helium cores, which is 0.4 mag fainter than the clump of old stars. Our models reproduce well this minimum, first observed by Corsi et al. (1994) using the clump against turn-off magnitude for LMC clusters (see Fig. 10 in Girardi \& Bertelli 1998). The models reproduce also the behaviour of the clump $I$-magnitude in clusters of ages from 2 to $10 \mathrm{Gyr}$, as measured by Udalski (1998). As expected (Fig. 1 in Girardi et al. 1998), in this age range the clump magnitude is almost constant.

The AGB-tip magnitude is mainly determined by the mass-loss rates adopted during this evolutionary phase. We have tested four different massloss prescriptions in our models (Girardi \& Bertelli 1998). The maximum AGB luminosity observed in LMC clusters of different ages, together with the initialfinal mass relation in the solar vicinity, allowed us to rule out some of the formulations. Models with Vassiliadis \& Wood's (1993) mass-loss provide the best results, describing well also the location of carbon stars in the $M_{\mathrm{bol}}$-age plane (Marigo et al. 1996), and the C-star luminosity functions in both Clouds (Marigo \& Girardi, this meeting). The latter models, including the formation of C-stars and the 'over-luminosity' caused by hot-bottom burning, are now being implemented in our codes.

The age distribution of LMC clusters reflects the occurrence of episodic cluster formation in this galaxy (Girardi et al. 1995). Thus, the information given by AGB stars in clusters is not complete over the entire age range of their progenitors (Marigo et al. 1996). Data on integrated $V-K$ colours of LMC clusters are more complete in age, and indicate that hot-bottom burning is probably operating for $M>3 M_{\odot}$ (Girardi \& Bertelli 1998); this is the simplest way to explain the lack of AGB stars (and hence blue colours) in clusters slightly older than $10^{8} \mathrm{yr}$. 


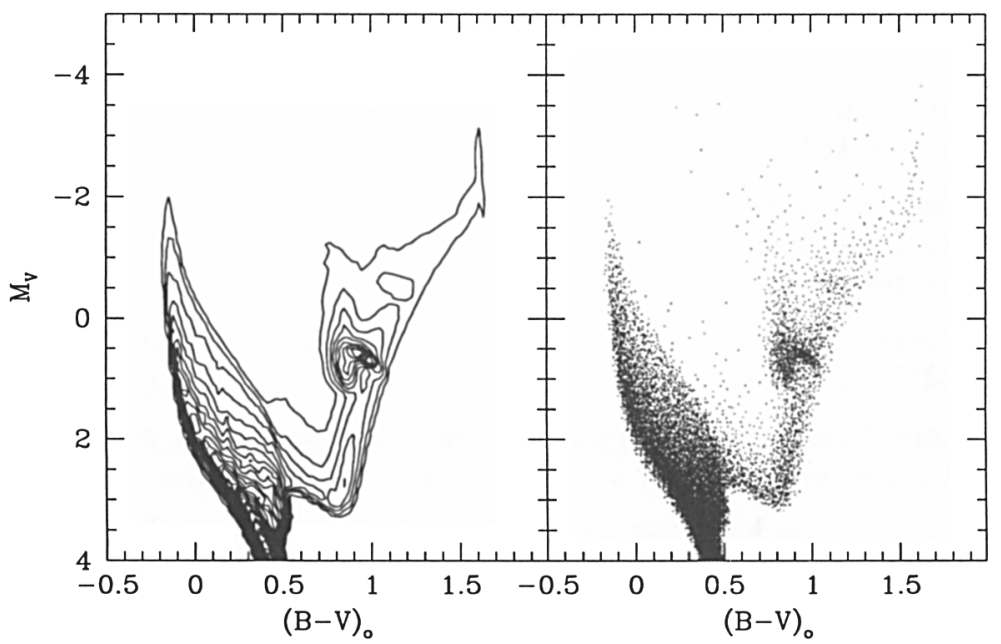

Figure 1. Theoretical distribution of stars in the $M_{V}$ vs. $B-V$ diagram, for a simple model meant to represent the LMC field. Left: Hess diagram, with contour levels at (density) ${ }^{1 / 2}$ intervals; Right: synthetic CMD with $2.5 \times 10^{4}$ stars.

Synthetic CMDs are being constructed for different histories of star formation and chemical enrichment. They reproduce well some fine details of observed CMDs (e.g., the width of the main sequence, the vertical extension of the red clump, the clump of early-AGB stars, the RGB bump), and contain TP-AGB stars in realistic proportions. Fig. 1 shows an example.

An interesting new feature in these models is the faint extension of the red clump. It has a solid theoretical basis, is present on the Hipparcos CMD of nearby stars (Girardi et al. 1998), and may correspond to the faint clumps recently observed in some fields of the LMC (Geisler et al., this meeting). A detailed description of this feature is provided in a forthcoming paper.

Acknowledgments. This work is funded by the Alexander von HumboldtStiftung. I thank the IAU for a travel grant to attend this meeting.

\section{References}

Bertelli, G., Bressan, A., Chiosi, C., et al. 1994, A\&AS, 106, 275

Corsi, C.E., Buonanno, R., Fusi Pecci, F., et al. 1994, MNRAS, 271, 385

Girardi, L., Chiosi, C., Bertelli, G., \& Bressan, A. 1995, A\&A, 298, 87

Girardi, L., \& Bertelli, G. 1998, MNRAS, 300, 533

Girardi, L., Groenewegen, M.A.T., Weiss, A., et al. 1998, MNRAS, 301, 149

Girardi, L., Bressan, A., Bertelli, G., \& Chiosi, C. 1999, in preparation

Marigo, P., Girardi, L., \& Chiosi, C. 1996, A\&A, 316, L1

Udalski, A. 1998, AcA, 48, 383

Vassiliadis, E., \& Wood, P.R. 1993, ApJ, 413, 641 


\section{Discussion}

Carme Gallart: Is the lower part of the RGB of your new tracks bluer than in the previous tracks?

Girardi: Yes, the present evolutionary tracks have a slightly bluer RGB than the previous Padova ones (Bertelli et al. 1994). This is due to the use of new tables of low-temperature opacities.

John Gallagher: Do your models reproduce the upper RGB in the Corsi et al. sample of MC star clusters?

Girardi: I don't remember exactly how the RGB compares with the observed ones. (I intend to present such a comparison in the next months.)

Jason Harris: Do you have any models that do not produce the faint extension of the Red Clump (since this feature is not observed in many fields)?

Girardi: No. All He-burning models slightly more massive than $2 \mathrm{M}_{\odot}$ burn helium at fainter luminosities. This behaviour can also be found in several other sets of evolutionary tracks in the literature. Notice that the faint clump should not be present in fields which did not form stars about 1 Gyr ago. 